\title{
Microscopic Polyangiitis With Positive Hantaan Virus-Specific Immunoglobuline M Antibody Caused by Cross Reaction: A Case Report
}

\author{
Diao ZONGLI, Zhang AIHUA, Wang GANG, Liu WENHU
}

Department of Nephrology, Beijing Friendship Hospital, Capital Medical University, Beijing, China

\begin{abstract}
Patients with microscopic polyangiitis may have an immune disorder and generate antibodies which may cross-react with foreign proteins. In this article, we report a 71-year-old female case of microscopic polyangiitis patient with positive Hantaan virus-specific immunoglobuline $M$ antibodies without hemorrhagic fever with renal syndrome. In this case, an immune disorder may generate antibodies that cross-reacting with Hantaan virus antigen, resulting in positive Hantaan virus-specific immunoglobuline $M$ antibody measurements.

Keywords: Cross reaction; Hantaan virus; hemorrhagic fever with renal syndrome; microscopic polyangiitis.
\end{abstract}

Hantaan virus infection may induce hemorrhagic fever with renal syndrome (HFRS), presenting with fever and acute kidney injury. ${ }^{1}$ Virusspecific immunoglobuline $\mathrm{M}$ (IgM) antibodies are important in the diagnosis of HFRS with a specificity of $96 \% .^{2}$ Microscopic polyangiitis (MPA) is an autoimmune disease characterized by positive myeloperoxidase anti-neutrophil cytoplasmic antibodies (MPO-ANCA). ${ }^{3}$ Patients with MPA may also have an immune disorder and present with several different specificities of antibodies which may cross-react with foreign proteins. ${ }^{4}$ However, few studies have been reported on cross-reactive antibodies in MPA patients in the literature. The current study describes a rare MPA case which was positive for Hantaan virus-specific IgM antibodies without HFRS.

\section{CASE REPORT}

A 71-year-old woman was admitted with fever, cough, and rapidly deteriorating kidney function.
The laboratory examinations showed increased serum creatinine levels from $323.7 \mu \mathrm{mol} / \mathrm{L}$ to $658 \mu \mathrm{mol} / \mathrm{L}$ for one month, while white blood cells $\left(9.8 \times 10^{9} / \mathrm{L}\right)$ and platelets $\left(249 \times 10^{9} / \mathrm{L}\right)$ were normal. Immunology test results revealed positive perinuclear ANCA, high titers of MPO-ANCA (236.47 $\mathrm{Ru} / \mathrm{mL}$, normal range $0-20 \mathrm{Ru} / \mathrm{mL}$ ), and negative proteinase 3-ANCA. Color Doppler ultrasound showed that the kidneys were normal in size, structure, and blood flow. Renal biopsy, during which 24 glomeruli were examined including four cellular, six fibrocellular, and one fibrous crescent, revealed necrotizing crescentic pauci-immune glomerulonephritis with fibrinoid necrosis in the capillary loops of some glomeruli. As a result, the patient was diagnosed with MPA. ${ }^{5,6}$

Since the patient presented with fever and acute kidney injury, and was previously exposed to rats which may transmit HFRS, indicating the diagnosis of HFRS was considered. We performed serological tests on day 60,61, 69 and 91 after the onset of the disease. Test results were positive

Received: August 11, 2013 Accepted: November 26, 2013 Published online: March 07, 2015

Correspondence: Liu Wenhu, M.D. Department of Nephrology, Beijing Friendship Hospital, Capital Medical University, 100050 Beijing, China.

Tel: +86-10-13901163354 e-mail: liuwenhu2013@163.com

@2015 Turkish League Against Rheumatism. All rights reserved. 
for Hantaan virus-specific IgM antibodies; whereas the colloidal gold-based immunochromatographic assay revealed negative results for virus-specific IgG antibodies. However, the patient did not experience typical phases of HFRS such as the febrile, hypotensive, oliguric, polyuric and convalescent phases. ${ }^{7}$ Moreover, white blood cells and platelet counts were normal, and virus-specific IgG antibodies remained negative, even on day 91 after the onset of the disease. These findings suggested that the patient did not have HFRS. Therefore, she was administered intravenous methylprednisolone pulse therapy, followed by methylprednisolone combined with cyclophosphamide. Finally, serum creatinine levels decreased to $137 \mu \mathrm{mol} / \mathrm{L}$ at 48 days after the start of treatment.

\section{DISCUSSION}

Patients with ANCA-associated systemic vasculitis (AAV) including MPA may have antibodies which cross-react with pathogen antigens, and may be involved in the pathogenesis of this disease. ${ }^{4}$ However, to the best of our knowledge, no direct evidence is available to support this. Recently, Kain et al. ${ }^{8}$ demonstrated a cross reactivity between AAV patient autoantibodies and the bacterial adhesive protein, FimH.

Our patient had Hantaan virus-specific IgM antibodies, as detected by the colloidal goldbased immunochromatography assay. In this test, Hantavirus recombinant antigen coated with colloidal is coated in the gold glass fiber paper. In case of serum containing specific IgM antibodies or other proteins which bind with Hantavirus recombinant antigen, the test would be positive. The test resulted positively for our patient, who also had an immune disorder, producing antibodies which cross-reacted with the hantavirus recombinant antigen.

In addition, our patient was positive for anti-thyroid peroxidase antibody (TPOAb) and anti-mitochondrial antibody type 2 (AMA-M2), however, did not have primary biliary cirrhosis (AMA-M2) or autoimmune thyroiditis (TPOAb) based on the clinical presentation and additional testing (data not shown). Therefore, we concluded that AMA-M2 and TPOAb may also be caused by cross reactions, indicating to our patient's immune disorder which may have generated cross-reactive antibodies.

In conclusion, MPA patients may be positive for Hantaan virus-specific IgM antibodies owing to cross reactivity, however, may not have HFRS. To the best of our knowledge, this is the first case demonstrating an association between MPA and Hantaan virus-specific IgM antibodies. We believe this study may contribute to the diagnosis of MPA patients who are positive for Hantaan virus-specific IgM antibodies, and enhance our understanding of MPA.

\section{Declaration of conflicting interests}

The authors declared no conflicts of interest with respect to the authorship and/or publication of this article.

\section{Funding}

The authors received no financial support for the research and/or authorship of this article.

\section{REFERENCES}

1. Krautkramer E, Zeier M. Old World hantaviruses: aspects of pathogenesis and clinical course of acute renal failure. Virus Res 2014;187:59-64.

2. Hujakka H, Koistinen V, Kuronen I, Eerikäinen P, Parviainen M, Lundkvist A, et al. Diagnostic rapid tests for acute hantavirus infections: specific tests for Hantaan, Dobrava and Puumala viruses versus a hantavirus combination test. J Virol Methods 2003;108:117-22.

3. Csernok E, Moosig F. Current and emerging techniques for ANCA detection in vasculitis. Nat Rev Rheumatol 2014;10:494-501.

4. Willcocks LC, Lyons PA, Rees AJ, Smith KG. The contribution of genetic variation and infection to the pathogenesis of ANCA-associated systemic vasculitis. Arthritis Res Ther 2010;12:202.

5. Jennette JC, Falk RJ, Bacon PA, Basu N, Cid MC, Ferrario F, et al. 2012 revised International Chapel Hill Consensus Conference Nomenclature of Vasculitides. Arthritis Rheum 2013;65:1-11.

6. Bosch X, Guilabert A, Font J. Antineutrophil cytoplasmic antibodies. Lancet 2006;368:404-18.

7. Chandy S, Abraham S, Sridharan G. Hantaviruses: an emerging public health threat in India? A review. J Biosci 2008;33:495-504.

8. Kain R, Exner M, Brandes R, Ziebermayr R, Cunningham D, Alderson CA, et al. Molecular mimicry in pauci-immune focal necrotizing glomerulonephritis. Nat Med 2008;14:1088-96. 